\title{
Cathodic Arcs: Fractal Voltage and Cohesive Energy Rule
}

\author{
André Anders ${ }^{a}$ \\ Lawrence Berkeley National Laboratory, Berkeley, California 94720, USA \\ Efim M.Oks, and Georgy Yu.Yushkov \\ High Current Electronics Institute, Russian Academy of Sciences, Tomsk, 634055, Russia
}

The noise of the burning voltage of cathodic arcs in vacuum was analyzed for a range of cathode materials (C, Mg, Ti, V, Ni, Cu, Zr, Nb, Ag, Hf, Ta, W, Pt, Bi, and Si). Cathodic arcs were generated in a coaxial plasma source, and the voltage noise was measured with a broadband (250 $\mathrm{MHz}$ ) measuring system. Each measurement of 50,000 points was analyzed by Fast Fourier Transform, revealing a power spectrum $\sim 1 / f^{2}$ for all metals over several orders of magnitude of frequency $f$ (brown noise). The absence of any characteristic time down to possible cutoffs at high frequencies supports a fractal model of cathode spots. Our preliminary research indicate that materials of high cohesive energy have a cutoff of self-similarity at $50 \mathrm{MHz}$ while no cutoff could be found up to $100 \mathrm{MHz}$ for non-refractory metals. The amplitude of colored noise scaled approximately linearly with the cathode's cohesive energy, which is another manifestation of the Cohesive Energy Rule.

a) electronic address: aanders@lbl.gov 
The plasma of cathodic vacuum arcs is produced at non-stationary cathode spots ${ }^{1}$. Much research, both theoretically ${ }^{2-7}$ and experimentally ${ }^{8-14}$, has been done for decades to understand the spot phenomena that produce non-equilibrium plasmas containing multiply ion charge states. The plasma is mesosonic ${ }^{15}$, i.e., subsonic for electrons and supersonic for ions, with respect to the local ion sound velocity. Difficulties in experimentation and modeling are associated with the spots' fast changes, small sizes, and extreme gradients of power density and particle densities. The formation of spot plasma is often described as a sequence of microexplosions, where cathode material transitions through various phases, from solid to liquid, non-ideal plasma and/or gas phases, finally turning into in a non-equilibrium, expanding, fully ion ionized plasma ${ }^{6,16}$.

With improvement of experimental techniques, faster and smaller phenomena have been discovered. This becomes especially striking when the issue of current density is considered. As the apparent area of electron emission was found to be smaller than previously thought, the corresponding current density increased accordingly ${ }^{17,18}$. There were arguments what constitutes an electron-emitting site. In one extreme, electron emission should occur from areas much larger than the molten crater due to intense ion bombardment of the crater vicinity. On the other hand, the most relevant electron emission area might be smaller than the later visible crater because crater formation could be the result of the action of several spot fragments or cells.

In order to make any useful statements on cathode processes and parameters of cathodic arc plasma, many researches decided to repeat measurements and average the measured data. For example, one can find data on average ion charge states ${ }^{10,19}$, average burning voltages ${ }^{20,21}$, average ion velocities ${ }^{15}$, or average ion velocity distribution functions ${ }^{22}$.

All parameters fluctuate with large amplitude; in some cases up to $100 \%$ of the average value, which can be associated with the explosive nature of cathode spot ignition and plasma production. While research focused on finding trends and models for averaged distributions and other data, only limited attention was paid to fluctuations or "noise," although the fluctuating signals can be expected to contain information on plasma formation processes. Fluctuations have been described in some publications ${ }^{1,12,23-26}$ but only few quantitative statements were made. Smeets and Schulpen ${ }^{12}$, looked at the noise of emitted light and ion fluxes. They noted a $1 / f$ decay of the ion flux noise up to a frequency $f \approx 15 \mathrm{MHz}$, followed 
by white noise at higher frequencies. Schülke and Siemroth ${ }^{27}$ observed that patterns of emitted light have self-similar properties, evident by using different optical magnification.

Among all physical quantities of cathodic arcs, the cathode fall voltage is special because it is intimately related to cathode processes, with very little distortion or superposition by secondary parameters of the system, such as the lifetime of excited levels in emission spectroscopy ${ }^{28}$. From experiments with variable electrode distance and movables probes it is known that most of the total arc voltage drops in the cathode fall; the remaining small drop depends on many conditions, such as anode size and geometry, presence of magnetic fields, etc. The arc voltage determines the energy input invested: while the arc current is determined by the impedance of the electric circuit, the arc voltage is self-adjusting to satisfy the energy need of the discharge, and in particular the cathode region where plasma is produced. This is the underlying idea of the empirically found Cohesive Energy Rule ${ }^{29}$, which states that the vacuum arc burning voltage depends approximately linearly on the cohesive energy of the material, $E_{C E}$. It was quantified as ${ }^{29}$

$$
V=V_{0}+a E_{C E},
$$

with $V_{0} \approx 14.3 \mathrm{~V}$ and $a=1.69 \mathrm{~V} /(\mathrm{eV} /$ atom $)$. Two arguments were brought forward to justify this empirical rule. First, the self-adjusting burning voltage connects properties of the plasma feedstock material with the energy conservation law. Second, many physical parameters show periodicity as expressed in the Periodic Table of the Elements, and therefore periodicity of the cohesive energy acts as a proxy for periodicity exhibited by other quantities such as melting and boiling temperatures or ionization energies.

Looking at the noise of arcs, a straightforward approach is to measure the noise of the total arc voltage, thereby characterizing the noise of the cathode fall, and ultimately the cathode processes causing it.

The noise of arcs was experimentally investigated by recording the burning voltage of a coaxial cathodic arc plasma source, and analyzing the data by Fast Fourier Transform (FFT). In order to preserve the frequency characteristics of the voltage noise, the arc source must be designed like a broad-band line. The easiest was to opt for a coaxial design of about $50 \mathrm{Ohm}$ impedance (Fig. 1). The center cathode was a rod of $6.25 \mathrm{~mm}$ diameter (1/4 inch), surrounded by a coaxial anode cylinder. On one end, the plasma source was essentially comprised of a high-current vacuum feedthrough. On the other end, a metal-coated 
alumina ceramic piece served as a holder to accommodate different cathode materials and as a means to initiate pulsed arcs via the self-triggering or "triggerless" method ${ }^{30}$.

The arc was fed by a large, $0.33 \mathrm{~F}$ capacitor bank and switched with a high-voltage, high-current transistor. The large capacity ensured that the arc current did not droop too much during the arc pulse. For most experiments, the arc had a current of $100 \mathrm{~A}$ for a duration of $2.5 \mathrm{~ms}$. When desired, the arc duration could be adjusted by the transistor switch. The current amplitude could be changed by the capacitor's charging voltage while keeping constant the current-limiting $2.0 \Omega$ resistor. The maximum voltage of the capacitor bank was $350 \mathrm{~V}$, which was supplemented a $600 \mathrm{~V}$ "kicker capacitor" for reliable arc initiation ${ }^{30}$. The arc repetition rate was 1 p.p.s. All arc experiments were done in oil-free vacuum at a base pressure of about $10^{-4} \mathrm{~Pa}$; no process gas was added.

The arc burning voltage was monitored directly at the vacuum feedthrough using a broadband 100:1 divider (Tektronix P5100, max. $250 \mathrm{MHz}$ analog). The divider was connected to a digital storage scope (Tektronix TDS744, max. $500 \mathrm{MHz}$ analog, $2 \mathrm{Gs} / \mathrm{s}$ ). Voltage data were captured in sample mode $(50,000$ data points per arc) with various time resolutions and their associated sample rates. The data were then exported from the scope's memory to a computer for further analysis.

FFT analysis results curves as shown in Fig. 2 for titanium. The most striking feature is the constant slope over several orders of magnitude, which is about $1 / f$ in amplitude spectrum and $1 / f^{2}$ in power spectrum. Occasionally, there are other features visible, like a little hump at the high-frequency end of some curves, but those features appear to be specific to a particular measurement or material. Another feature of FFT curves is the broad band of data, especially noticeable at the high-frequency end. To better evaluate FFT data from here on, 16 curves obtained for constant measuring conditions were averaged. It should be emphasized that this averaging is done after FFT, thus information on noise is not lost.

There are a number of interesting questions that need to be addressed to further learn about cathode processes. Among them are: What is the influence of arc current and cathode material on the noise of arcs? How does the noise relate the elementary cathode processes?

In a first step, the influence of the arc current was measured. In the current range from 50 A to 240 A it was found that the FFT curves did not change their character but shifted slightly: the higher the current the lower the noise amplitudes. The shifts are barely visible in log-log presentation. 
The next step was to measure whether arcs on cathode materials other than titanium give the same of similar FFT curves. The arc plasma source was designed for exchangeable cathode materials. To limit the parameter space, the following experiments were done with a constant arc duration of $2.5 \mathrm{~ms}$, constant arc current of $100 \mathrm{~A}$, and constant time resolution of $100 \mathrm{~ns} / \mathrm{div}$ ( $2 \mathrm{~ns}$ per data point, or $500 \mathrm{kS} / \mathrm{s}$ ), with a total acquisition time of $100 \mu$ s per measurement. A wide range of different cathode materials was selected, including C, Mg, Ti, V, Ni, Cu, Zr, Nb, Ag, Hf, Ta, W, Pt, Bi, and Si. Each of these elements gave FFT curves with approximately the same slope of $1 / f$ (spectral amplitude vs. frequency) and $1 / f^{2}$ (spectral power vs. frequency) in log-log presentation. However, each material has its own characteristic level of noise amplitude, resulting in a different position of the curve. Figure 3 shows curves of two extreme materials, Bi and W. For comparison, the FFT procedure was also applied to the voltage zero line (i.e., no arc burning). Interestingly, the zero-line gives about the same slope at relatively low frequency and white noise at higher frequencies, which will be further discussed below.

To quantify the material-dependent noise amplitude, a frequency of $40 \mathrm{MHz}$ was arbitrarily picked and the amplitude difference between FFTs of zero line and arc noise was determined. The selection of this frequency is not critical as long as it is in the physically meaningful range. At too low frequencies, data are scare, and at too high frequencies, the FFT curves approach the FFT of the zero line, i.e. there is no arccaused noise signal anymore. The differences at $40 \mathrm{MHz}$ were normalized with the respect to the largest difference observed, which was for tungsten. The result is plotted against the cohesive energy of the cathode material, as shown in Fig. 4.

These measurements deserve critical interpretation. First, it is remarkable that for all materials investigated, FFT curves have a constant slope (in log-log presentation) over several orders of magnitude. The well-known theory of colored noise $\mathrm{e}^{31,32}$ is based on power laws

$$
P(f) \sim 1 / f^{\alpha},
$$

where $P$ is the spectral power and $\alpha$ an exponent determining the "color" of the noise. The noise is white for $\alpha=0$, i.e. independent of frequency; pink for $\alpha=1$, typical for systems of self-organized criticality ${ }^{33}$; brown for $\alpha=2$, for systems exhibiting large-scale fractal stochastic (Brownian) motion ${ }^{31}$; and black for $\alpha>2$, occurring in self-similar systems with positive feedback, like the stock market ${ }^{32}$. 
In a recent work ${ }^{34}$ it was proposed that arc cathode spots are stochastic fractal objects that lack scaling over a wide range of values in space and time. It is clear that physical cutoffs exist but the (rather elusive) characteristic elementary size and times have not been confirmed despite numerous sophisticated investigations ${ }^{13,35,36}$. Since the early work by $\mathrm{Kesaev}^{9}$, the existence of spot cells or fragments was confirmed by high-resolution diagnostics ${ }^{13,37}$, though the lower scaling cutoff would only be confirmed if higher resolution techniques did not find smaller structures and faster changes.

Our measurements (Fig. 3) indicate that there is no cutoff of self-similarity in the fractal voltage until one approaches the region of $50 \mathrm{MHz}$ and faster (or inverse, $20 \mathrm{~ns}$ and shorter). There are deviations from the power law, Eq. (2), for materials of high cohesive energy (e.g. Ta, W). At this time it is not clear whether these deviations are true features of cathode processes, or, for example, plasma instabilities unrelated to cathode processes. Further research is needed to address this issue.

The slight reduction of noise with increasing current can readily be explained by the superposition of short-lived elementary processes: fragments, cells, or ectons in Mesyats' terminology ${ }^{16}$. Superposition would reduced the expected standard deviation of a measured stochastic signal as $\sigma \sim 1 / \sqrt{n}$, where $n$ is the number of active emission sites. The same argument was brought forward to explain a reduction in noise of collected ion current when the arc current was enhanced ${ }^{38}$.

Interestingly, even the zero-line itself showed power-law (fractal) behavior, at least at low frequencies. This is not unexpected because the superposition of small elementary events causes larger fluctuations at lower frequency ${ }^{39}$. At high frequencies, white noise is observed, which is associated with the small-amplitude cutoff given by the digitizing depth of the digital oscilloscope.

With these considerations one may return to the physical picture of arc cathode processes and conclude that they are comprised of fast, small-scale events of electron emission and plasma production, which are sufficiently fast that all observation at longer times lack characteristic scale, indicating selfsimilar, fractal behavior. The observed spectral power is proportional to $1 / f^{2}$, which is brown noise. It is interesting to contemplate that the brown noise found by time series analysis is associated with twodimensional spatial Brownian motion on the cathode surface. Brownian motion is known to be the scaling limit of Random Walk for vanishing step length, and therefore Brownian motion is often approximated by random walk models to make computation more efficient. Random walk of cathode spots was extensively 
investigated $^{40,41}$ and a fractal dimension of 2 (characteristic for Brownian motion) was experimentally found by analyzing arc $\operatorname{traces}^{42}$.

Thus, the present arc voltage noise measurements support a fractal model of cathode spots for all materials, with possible scaling cutoffs at $50 \mathrm{MHz}$ or higher frequency. The preliminary data show that cathode materials of higher cohesive energy have a lower cutoff frequency than material with small cohesive energy, indicating that the emission current per fragment or emission site is higher for materials of high cohesive energy. Peaks observed for $f>50 \mathrm{MHz}$, could be a signature of fast elementary emission processes $\left(\right.$ ectons $^{5}$ ), though that conclusion remains to be confirmed. Materials of low cohesive energy have shorter elementary times, hence self-similar scaling cutoff are not easily observed. The amplitude frequency spectrum of arc voltage was found to be an approximate linear function of the cathode's cohesive energy, which is a manifestation of the Cohesive Energy Rule.

\section{Acknowledgements}

The authors thank Robert MacGill for technical assistance in fabricating the arc plasma source. This work was supported by the U.S. Department of Energy, Initiatives of Proliferation Prevention, project IPP-LBNL-T2-196, under Contract No. DE-AC03-76SF00098, and by a Grant of the President of the Russian Federation, the Young Dr. Sci. Program MD-148.2203.02. 


\section{References}

Martin, and D. M. Sanders (Noyes, Park Ridge, N.J., 1995), p. 208.

5

8

10

A. Anders, B. Yotsombat, and R. Binder, J. Appl. Phys. 89, 7764 (2001).

E. Byon and A. Anders, J. Appl. Phys. 93, 1899 (2003). (1990).

V. M. Lunev, V. G. Padalka, and V. M. Khoroshikh, Instrum. Exp. Tech. 19, 1465 (1976).

I. G. Brown, P. Spädtke, D. M. Rück, and B. H. Wolf, Nucl. Instrum. Meth. Phys. Res. A 295, 12 

(2005). (1998).

V. F. Puchkarev, J. Phys. D: Appl. Phys. 24, 685 (1991).

A. Anders, IEEE Trans. Plasma Sci. 27, 1060 (1999). T. Schülke and P. Siemroth, IEEE Trans. Plasma Sci. 24, 63 (1996).

S. Anders, A. Anders, and B. Jüttner, J. Phys. D: Appl. Phys. 25, 1591 (1992).

A. Anders, in Emerging Applications of Vacuum-Arc-Produced Plasma, Ion and Electron Beams, edited by I. Brown and E. Oks (Kluwer Academic Publishers, Dordrecht, 2002), p. 1.

A. Anders, I. G. Brown, R. A. MacGill, and M. R. Dickinson, J. Phys. D: Appl. Phys. 31, 584

A. R. Osborne and A. Pastorello, Phys. Lett. A 181, 159 (1993).

M. Schroeder, Fractals, Chaos, Power Laws: Minutes from an Infinite Paradise, 8th Ed. ed. (W.H.Freeman and Company, New York, 2000).

P. Bak, C. Tang, and K. Wiesenfeld, Phys. Rev. Lett. 59, 381 (1987).

A. Anders, IEEE Trans. Plasma Sci. 33, submitted for Special Issue (2005).

B. Jüttner, J. Phys. D: Appl. Phys. 14, 1265 (1981).

S. A. Barengolts, G. A. Mesyats, and D. L. Shmelev, IEEE Trans. Plasma Sci. 31, 809 (2003).

B. Jüttner, J. Phys. D: Appl. Phys. 31, 1728 (1998).

A. Anders, K. Fukuda, and G. Y. Yushkov, J. Phys. D: Appl. Phys 38, accepted for publication

D. Spasojevic, S. Bukvic, S. Milosevic, and H. E. Stanley, Phys. Rev. E 54, 2531 (2001).

E. Hantzsche, B. Jüttner, and H. Pursch, J.Phys.D: Appl.Phys. 16, L173 (1983).

J. E. Daalder, J.Phys.D: Appl.Phys. 16, 17 (1983).

S. Anders and B. Jüttner, IEEE Trans. Plasma Sci. 19, 705 (1991). 


\section{Figure Captions}

Fig. 1 Schematic of the coaxial plasma source used for the voltage noise experiments.

Fig. 2 Example of FFT of voltage data for a 50 A titanium arc, captured with different time resolution at the oscilloscope (5 ns/div, $1 \mu \mathrm{s} / \mathrm{div}, 100 \mu \mathrm{s} / \mathrm{div})$, where each division has 50 data points.

Fig. 3 FFT curves (average of 16 amplitude vs. frequency measurements) for two extreme materials, Bi and W. For comparison, the same FFT procedure was also applied to the voltage zero line (i.e., no arc burning). All arc and analysis conditions were identical, except the cathode material: $100 \mathrm{~A}, 500 \mathrm{kS} / \mathrm{s}$, for $100 \mu$ s per measurement.

Fig. 4. Amplitude difference between FFT curves of arc voltage and FFT of the voltage zero line, measured at $40 \mathrm{MHZ}$ and under conditions like in Figure 3, normalized with respect to the difference for tungsten and plotted versus the cohesive energy of the cathode material. 


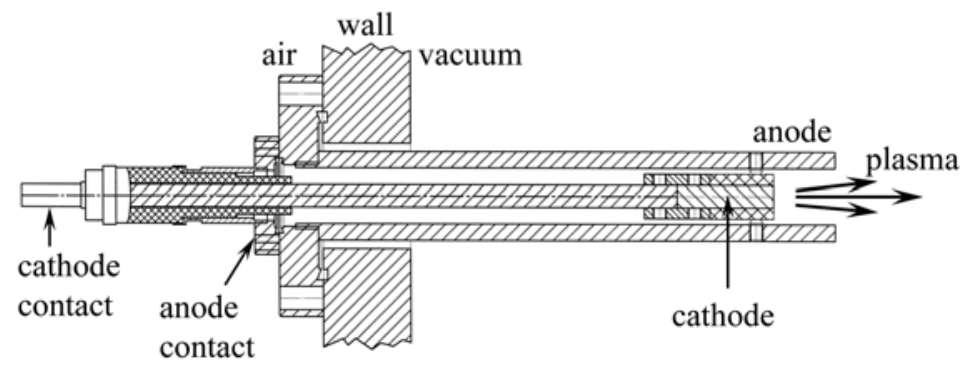

Fig. 1 


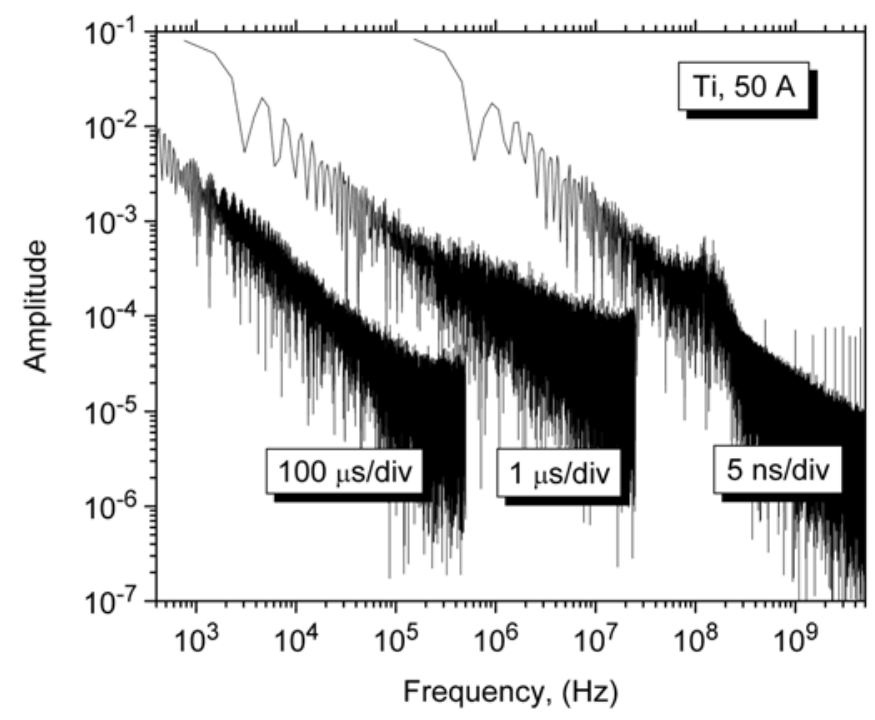

Fig. 2 




Fig.3 


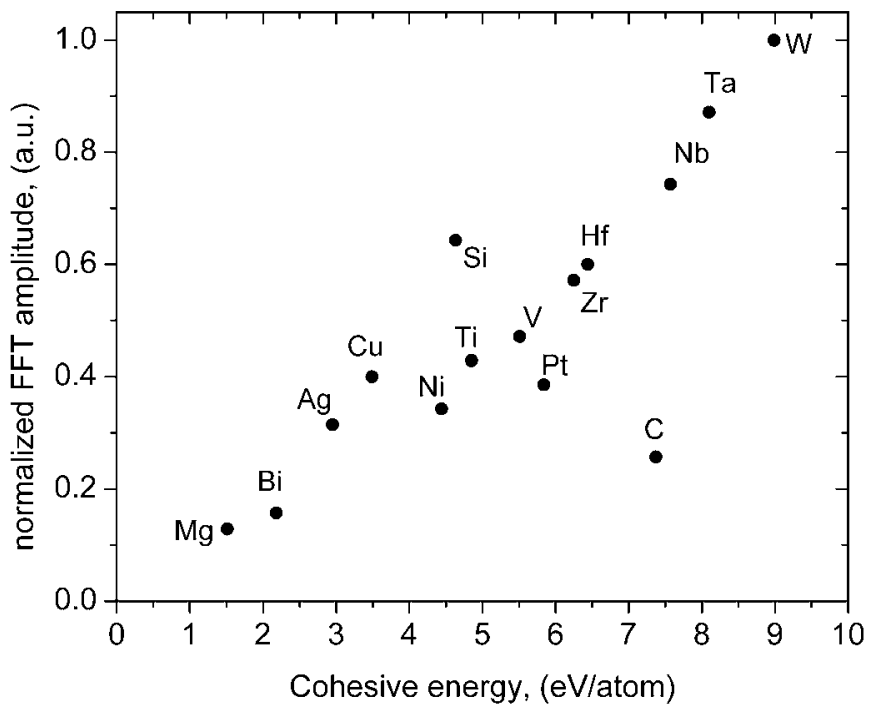

Fig. 4 